\title{
EOPs: Estrategias para discontinuar el consumo de benzodiacepinas en personas ancianas
}

\author{
Strategies for discontinuing benzodiazepine use in the elderly
}

Facundo de Ciervo*

\begin{abstract}
Resumen
Un médico de familia se pregunta sobre los riesgos y los beneficios del consumo de benzodiacepinas en una paciente anciana de 76 años, y se plantea cómo ayudarla en la discontinuación de la droga para prevenir futuras caídas. Luego de formular la pregunta y realizar la búsqueda bibliográfica se incluyeron dos metanálisis de los cuales se desprende que la tasa de discontinuación de las benzodiacepinas aumenta en quienes reciben estrategias breves de consejería, estrategias de reducción gradual de la dosis, e intervenciones psicológicas de apoyo.

\section{Abstract}

A family physician ask himself about the risks and benefits of the use of benzodiazepines in an elderly patient of 76 years, and considers how to help her to discontinue this medication to prevent future falls. After elaborating an appropriate question and performing a literature search, two meta-analyzes were included. These shows that the rate of discontinuation of benzodiazepines increases in those receiving brief counseling strategies, strategies pointing to gradual dose reduction, and psychological support interventions.
\end{abstract}

De Ciervo F. Estrategias para discontinuar el consumo de benzodiacepinas en personas ancianas. Evid Act Pract Ambul Oct-Dic 2013; 16(4):155-157.

\section{Escenario clínico}

Un médico de familia atiende a una mujer de 76 años viuda y que vive sola. Tiene diagnóstico de hipertensión arterial, tratada con $10 \mathrm{mg} /$ día de enalapril y desde que falleció su marido, hace cinco años, consume 0,5 mg/día de alprazolam para dormir. Realiza todas las actividades de la vida diaria y refiere haberse caído desde su propia altura en dos oportunidades durante los últimos tres meses mientras realizaba actividades de limpieza doméstica.

No tiene otros antecedentes médicos personales relevantes. El médico de familia se pregunta sobre los riesgos y los beneficios del consumo de benzodiacepinas en esta paciente, y se plantea cómo ayudarla en la discontinuación de la droga para prevenir futuras caídas.

\section{Pregunta que generó el caso}

En pacientes ancianos (mayores de 65 años de edad) que consumen benzodiacepinas crónicamente y que no tienen una indicación terapéutica específica para la su continuación (población), ¿Cuál es la estrategia más efectiva (intervención) para la discontinuación del uso de benzodiacepinas? (resultado)

\section{Estrategia de búsqueda y selección de artículos}

Se realizó una búsqueda en Pubmed, TripDataBase y Cochrane con las palabras claves "benzodiazepine" AND "discontinuation" y "benzodiazepine" AND "withdrawal". Se restringió la búsqueda a meta-análisis y revisiones sistemáticas, artículos realizados en humanos publicados en inglés o español, y que hayan incluido población anciana.

Fueron identificados tres meta-análisis que procuraron responder la pregunta clínica planteada al comienzo: Parr y col. ${ }^{1}$, Oude Voshaar y col. ${ }^{2}$ y Mugunthan $\mathrm{K} \mathrm{y} \mathrm{col.}{ }^{3}$, Se eligieron el de Parr y col. y Mugunthan y col. por haberse publicado en los últimos cinco años, y por haber incluido sólo pacientes del ámbito ambulatorio y ancianos.

Algunas consideraciones respecto al consumo de benzodiacepinas en ancianos

Las benzodiacepinas son uno de los fármacos más consumidos. Son utilizadas para trastornos de ansiedad, interrupción del consumo de alcohol, como terapia adyuvante para la esquizofrenia y la depresión y como relajantes musculares. Los beneficios que derivan de su uso a corto plazo son indudables, pero su uso a largo plazo puede producir somnolencia diurna, alteración de los reflejos, deterioro cognitivo, aumento del riesgo de caídas y fracturas en ancianos, y dependencia. La mayoría de las guías recomiendan restringir su uso a tres o cuatro semanas pero muchos pacientes las consumen durante años debido a la adicción que provocan, incluso a bajas dosis ${ }^{4}$. Existe evidencia de que el consumo de benzodiacepinas, así como el de otros psicofármacos está fuertemente vinculado a un aumento de la probabilidad de caídas ${ }^{5}$. La problemática de las caídas está involucrada en aproximadamente el $10 \%$ de las visitas a las centrales de emergencia y el $6 \%$ de las hospitalizaciones de urgencia de personas ancianas; e independientemente de otras condiciones de salud, la persona con caídas frecuentes suele restringir sus actividades de la vida diaria y tiene una mayor probabilidad de ser institucionalizada.

Los intentos de interrupción de las benzodiacepinas pueden causar ansiedad anticipatoria, insomnio de rebote, irritabilidad y otros síntomas que perpetúan el espiral de dependencia y abuso; logrando sólo el 6\% de los individuos abandonar espontáneamente su uso 5 .

Dado que muchos de los tratamientos con benzodiacepinas son iniciados en el ámbito de la atención primaria, los profesionales que trabajamos en dicho ámbito deberíamos cumplir un rol importante en la limitación de su duración y su interrupción. Vale destacar que las intervenciones para discontinuar el consumo de benzodiacepinas han sido evaluadas en varios estudios y que luego de una reducción gradual de la dosis, las tasas de abandono exitoso a los 12 meses van de 24 a $51 \%$.

\section{Resumen de la evidencia}

1) Parr $J$ y col. Effectiveness of current treatment approaches for benzodiazepine discontinuation: a meta-analysis. Addiction 2009; 104(1): 13-24

\section{Objetivo}

Evaluar la efectividad de los abordajes terapéuticos actuales para la discontinuación de benzodiacepinas en pacientes ambulatorios.

\section{Diseño}

Revisión sistemática.

\footnotetext{
* Alumno de la Escuela de Medicina del Instituto Universitario del Hospital Italiano de Buenos Aires.

$\S$ Más de un tercio de las personas mayores de 65 años sufren caídas cada año, que en la mitad de las personas son recurrentes. Aproximadamente una de cada diez caídas resultan en daños serios como fracturas de cadera, hematomas subdurales, daños serios de tejidos blandos o heridas en la cabeza.
} 


\section{Criterios de búsqueda}

Fuentes: PsycLIT (1840-2005), MEDLINE (1966-2005) y EMBASE (1990-2005). La búsqueda se extedió hasta 2007. Palabras clave: (abuse or dependen* or addiction or overuse or misuse or chronic or long-term or cessation or withdraw* or reduc $^{*}$ or discontinu* or taper* or cutting) AND (benzodiaz* or nitrazepam or temazepam or triazolam or flunitrazepam or midazolam or zopiclone or zolpidem or oxazepam or alprazolam or diazepam or lorazepam or clobazam or bromazepam or

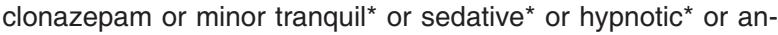
xiolytic* or psychotropic*) AND (random* or RCT).

\section{Criterios de selección de artículos}

Se seleccionaron Ensayos Clínicos Controlados Aleatorizados (ECCA) que hubieran evaluado la efectividad de los tratamientos para la discontinuación del uso de benzodiacepinas. Dos revisores seleccionaron los estudios independientemente y resolvieron los desacuerdos con un tercer revisor.

Los estudios fueron incluidos si habían comparado tratamientos adyuvantes para la discontinuación de benzodiacepinas con la práctica habitual de atención ${ }^{\star *}$ o con la reducción gradual de la dosis en participantes ambulatorios que hubieran consumido estas drogas durante tres meses o más. Para ser incluidos en el metanálisis, los estudios debían reportar datos suficientes que permitieran calcular las tasas de discontinuación en un análisis por intención de tratar.

\section{Resultados de la revisión}

Fueron seleccionados 32 ECCA que habían incluido participantes de 38 a 71 años. La duración media del uso de benzodiacepinas fue de dos a 19 años. Calidad de los estudios: Algunas de las limitaciones incluyeron: definición inadecuada de dependencia, seguimiento a corto plazo (la mayoría fue menor a 12 meses), falta de "ciego" y de evaluación indepen'diente de los resultados ${ }^{+1}$.

\section{Abordajes terapéuticos evaluados por los estudios}

a) Práctica habitual de atención (PHA) vs. intervenciones breves; b) PHA vs. reducción gradual de la dosis (RGD); c) PHA vs. intervenciones psicológicas (IP); d) RGD vs. RGD más IP; e) RGD vs. RGD más farmacoterapias sustitutivas; f) IP más discontinuación gradual o abrupta.

\section{Resultados por grupo de intervenciones evaluadas}

Comparadas con la práctica habitual de atención, tanto las intervenciones breves, como las reducciones graduales de las dosis y las intervenciones psicológicas se asociaron a un aumento significativo de la discontinuación de las benzodiacepinas.

Práctica habitual de atención vs. intervención breve ${ }^{\ddagger}$ : En los tres estudios que habían aleatorizado individuos $(n=532)$ se observó una tendencia favorable a los asignados a intervenciones breves (OR 4,37; IC95\% 2,28 a 8,40), mientras que en los dos estudios que habían aleatorizado profesionales
Práctica habitual vs. reducción gradual de la dosis $(n=107)^{\dagger t t}$ : La reducción gradual de la dosis fue más efectiva que la PHA (OR 5,96; 2,08 a 17,11).

Práctica habitual de atención vs. intervenciones psicológicas $^{* * *}$ : Las intervenciones psicológicas sumadas a la reducción gradual de la dosis fueron más efectivas que la PHA (OR 3,38; $1,86$ a 6,$12 ; n=354)$; mientras que un único estudio que realizó un seguimiento prolongado de los participantes de la investigación documentó que las intervenciones psicológicas se asociaron a beneficios duraderos (OR 13,5; 1,20 a 152,21; $n=20$ ). Reducción gradual de la dosis con o sin el agregado de tratamiento psicológico adicional : Desde el día de la aleatorización transcurrieron un promedio de 49 días (rango de 6,5 a 84) hasta el abandono de la medicación. Cuando se lo comparó con la reducción gradual de la dosis utilizada como única herramienta terapéutica, el añadido de tratamiento psicológico adicional aumentó en forma leve pero estadísticamente significativa las tasas de discontinuación en los siete estudios evaluados (OR 1,82; 1,25 a 2,67; $n=454$ ); hallazgos concordantes con los de los seis estudios que realizaron seguimientos más prologados (OR 1,88; 1,19 a 2,97; $\mathrm{n}=308$ ).

Reducción gradual de la dosis con o sin el agregado de farmacoterapia sustitutiva: Los pacientes demoraron un promedio de 36 días (rango 14 a 70 ) en abandonar las benzodiacepinas. Los 14 estudios evaluados no lograron detectar diferencias clínicamente relevantes ( $n=927$ ).

Tratamiento psicológico más discontinuación gradual o abrupta: No se encontraron diferencias significativas en las tasas de discontinuación a corto ni a mediano plazo.

\section{Conclusiones}

En comparación con la práctica habitual, la tasa de discontinuación de las benzodiacepinas fue mayor en quienes recibieron consejo mediante intervenciones breves (con o sin intervenciones más extensas); mientras que la estrategia de reducción gradual de la dosis sumada a intervenciones psicológicas podría aportar beneficios más duraderos. Respecto del uso de farmacoterapia sustitutiva, la evidencia actual es insuficiente como para respaldar su indicación.

2) Mugunthan K, McGuire T, Glasziou P. Minimal interventions to decrease long-term use of benzodiazepines in primary care: a systematic review and meta-analysis. British Journal of General Practice 2011; 61(590): e573-e578

\section{Objetivo}

Evaluar la efectividad de intervenciones mínimas en atención primaria para reducir la utilización crónica de benzodiacepinas en adultos.

Diseño

Meta-análisis

\section{Búsqueda y selección de artículos}

Cochrane Central Register of Controlled Trials (CENTRAL),

** La práctica habitual de atención (en inglés: "routine care") fue definida de manera distinta en los distintos ensayos, aunque por lo general se trató de un grupo control que fue informado respecto de la aleatorización, pero que no recibió la intervención estudiada en la otra rama de estudio.

† Si bien los ensayos clínicos seleccionados tuvieron tiempos de seguimiento disímiles y diferentes puntajes de calidad, no se documentó correlación entre el puntaje de la calidad y el tamaño del efecto evaluado.

¥¥ Intervención breve: el médico de cabecera enviaba una carta a los pacientes que hubieran repetido sus prescripciones de benzodiacepinas durante tres meses o más. En dicha carta se recalcaba la necesidad de suspender el uso de estos fármacos y algunos estudios sumaron un folleto de "autoayuda" a este tipo de intervención.

t+† Intervenciones psicológicas: entrenamiento en relajación, psicoeducación para el abandono de las benzodiacepinas y enseñanza de estrategias para abordar el insomnio.

${ }^{\star * *}$ Tratamiento psicológico adicional: entrenamiento en relajación, tratamiento cognitivo-conductual para el insomnio, y un programa de múltiples componentes relacionado al manejo del abandono de las benzodiacepinas y de la ansiedad. 
MEDLINE y EMBASE hasta 2010. Fueron seleccionados ECCA que habían evaluado la eficacia de intervenciones mínimas (cartas, información, consulta breve en atención primaria, consejería) para la discontinuación del uso crónico (tres meses o más) de benzodiacepinas en adultos. Todos los estudios fueron realizados en el Reino Unido y reportaron un seguimiento de seis meses. Dos revisores independientes seleccionaron los estudios.

\section{Medición de resultados principales}

Resultado primario: utilización de benzodiacepinas medida por registros de prescripción. Resultados secundarios: estado general de salud medido por cuestionario general de salud, GHQ o la versión corta del SF-36.

\section{Resultados}

Fueron incluidos tres ECCA con un total de 615 participantes (60\% mujeres y edad promedio 60 años). Se observó una disminución significativa en el consumo de benzodiacepinas en los grupos asignados a intervenciones mínimas vs cuidado usual (RR 2,04; IC95\% 1,48 a 2,83). Se observó un aumento significativo en la cesación en el grupo intervención (RR 2,31; $1,29$ a 4,17$)$

\section{Conclusión de los autores}

Las intervenciones breves realizadas por médicos de atención primaria para la discontinuación del uso crónico de benzodiacepinas constituyen una estrategia efectiva para disminuir o suspender su uso. No fueron analizadas las consecuencias adversas de la disminución del consumo. Las conclusiones deben interpretarse con cautela debido a la escasa evidencia y a la calidad variable de los estudios.

\section{Comentarios finales y recomendaciones}

Volviendo al caso que motivó esta búsqueda bibliográfica, los estudios evaluados en ambas revisiones sistemáticas incluyeron personas adultas y ancianas, por lo que sus conclusiones podrían trasladarse a nuestra paciente en cuestión.

Si bien resulta difícil responder cuál es la mejor estrategia de discontinuación, contamos con datos consistentes para apoyar la realización de distintas intervenciones para la discontinuación de benzodiacepinas, particularmente a través de la reducción gradual de la dosis, con o sin intervenciones psicológicas adicionales. También resultaron efectivas las intervenciones breves que emplearon cartas de recomendación de la discontinuación o folletos de auto-ayuda para el manejo del insomnio.

Las tasas de discontinuación del uso de las benzodiacepinas son alentadoras y pueden derivan en un enorme beneficio para la salud de los ancianos que no tienen una indicación precisa para su uso, principalmente en aquellos con elevado riesgo de caídas.

\section{Referencias}

1. Parr J y col. Effectiveness of current treatment approaches for benzodiazepine discontinuation: a meta-analysis. Addiction 2009; 104(1): 13-24.

2. Oude Voshaar R y col. Strategies for discontinuing linf-term benzodiazepine use: meta-analysis. Br J Psychiatry 2006; 189:213-20.

3. Mugunthan $\mathrm{K}$ y col. Minimal interventions to decrease long-term use of benzodiazepines in primary care: a systematic review and meta-analysis. British Journal of General Practice 2011; 61(590): e573-e578.

4. Vicens $C$ y col. Withdrawal from long-term benzodiazepine use: randomised trial in family practice. Br J Gen Pract 2006; 56:958-63.

5 Tinetti ME. Preventing Falls in Elderly Persons. N Engl J Med 2003; 348:42-49. 